\title{
Ammissibilità della prova e divieti probatori
}

\author{
Admissibility of evidence and exclusionary rules
}

Paolo Ferrua $^{1}$

Università degli Studi di Torino/Italia

paolo.ferrua@unito.it

https://orcid.org/0000-0002-7459-6762

\begin{abstract}
AвStract: Prova 'ammissibile' è tutto ciò che può essere legittimamente valutato in ordine a una proposizione da provare. Prova 'inammissibile' è quella che il giudice non ha il potere di assumere e, come tale, appartiene alla sfera del giuridicamente irrilevante. Occorre tenere ben distinte le questioni relative all'ammissibilità della prova da quelle relative all'efficacia probatoria, ossia alla persuasività. Analogamente non va confusa la prova come premessa probatoria, che esprime una mera potenzialità (la prova su $x$ ) con la prova come risultato (la prova di $x$ ), che indica un esito positivo. Con riguardo alle premesse probatorie, resta fondamentale la controversa distinzione tra prove dichiarative e prove critico-indiziarie. Parlando di prove «acquisite in violazione dei divieti stabiliti dalla legge», l'art. 192 c.p.p. è stato variamente interpretato: secondo alcuni, va riferito alle sole prove oggetto di un divieto probatorio, ossia inammissibili; secondo altri, anche alle prove 'ottenute' attraverso una qualsiasi violazione della legge (penale processuale o sostanziale o persino civile). Di recente è stata sollevata una questione di legittimità sul presupposto che le prove assunte in spregio dei diritti costituzionalmente tutelati non possano essere utilizzate, anche in assenza di un esplicito divieto probatorio. La Corte costituzionale ha, tuttavia, dichiarato inammissibile la questione con argomenti poco convincenti. Si è così persa la preziosa occasione per il definitivo chiarimento di un fondamentale interrogativo.

PAROle ChIAVE: prova; divieti probatori; prova dichiarativa; prova indiziaria; prova atipica; prova neuroscientifica; libertà di autodeterminazione; diritti fondamentali; Costituzione.
\end{abstract}

1 Professore Emerito di Procedura penale, Università degli Studi di Torino/Italia. 
ABStRAct: 'Admissible' evidence is anything that can be legitimately evaluated about a proposition to be proved. 'Inadmissible' evidence is the one that the judge does not have the power to undertake and, as such, belongs to the sphere of the legal irrelevancy. Issues related to the evidence admissibility must be distinct from those regarding to evidence efficacy, ie persuasiveness. Equally, there must not be confusion between the evidence as a probative premise, which states a mere potentiality (the evidence on $\mathrm{x}$ ), and the evidence as a result (the evidence of $\mathrm{x}$ ), which indicates a positive outcome. Concerning the probative premises, the controversial distinction between declarative evidence and critical-circumstantial evidence remains fundamental. Observing the evidence "obtained in violation of the prohibitions established by law", the art. 192 c.p.p. has been interpreted in various ways: according to some, it refers only to the evidence object of an exclusionary rule, ie inadmissible; according to others, even the evidence 'obtained' through any violation of the law (criminal, procedural or substantive, or even civil). A question of legitimacy has recently been raised on the statement that evidence taken in violation of constitutionally protected rights cannot be used, even in the absence of an explicit exclusionary rule. The Constitutional Court has, however, declared the question inadmissible with unconvincing arguments. Consequently, the precious opportunity for the definitive clarification of a fundamental question was lost.

KeYwords: evidence; exclusionary rules; declarative evidence; circumstantial evidence; atypical evidence; neuroscientific evidence; freedom of selfdetermination; fundamental rights; Constitution.

\section{IL CONCETTO DI PROVA}

Il tema dell'ammissibilità della prova attiene al primo elemento della terna che compone l'operazione probatoria, nella quale occorre distinguere: $a$ ) le premesse o proposizioni probatorie o prove in senso proprio; $b$ ) la proposizione da provare, vale a dire l'oggetto della prova; c) l'atto del provare, connotato dalla regola dell'oltre ogni ragionevole dubbio, che congiunge le prime alla seconda ${ }^{2}$.

2 Sulla struttura triadica dell'operazione probatoria, v. P. Ferrua, La prova nel processo penale, vol. I, Struttura e procedimento, 2 ed., Giappichelli, Torino, 2017. 
Prova 'ammissibile' nel processo penale è ogni dato che possa essere legittimamente acquisito e valutato dal giudice in ordine ad una determinata proposizione da provare. Di qui, per differenza, la nozione di prova 'inammissibile' come la prova che il giudice non ha il potere di acquisire al processo, in quanto oggetto di un divieto probatorio, ossia di una regola di esclusione ${ }^{3}$. Mancando il potere, la prova illegittimamente acquisita è giuridicamente irrilevante, quindi inutilizzabile a fini decisori; né occorre che la legge preveda espressamente l'invalidità processuale, essendo in re ipsa che l'atto compiuto in assenza del potere sia giuridicamente inesistente ${ }^{4}$.

Diverso il discorso per l'inosservanza delle modalità previste per l'assunzione di una prova in sé ammissibile. Qui l'atto - compiuto in presenza del potere, ma in violazione di un dovere - lungi dall'essere giuridicamente inesistente, è affetto dal vizio tassativamente previsto dalla legge (nullità o altra sanzione: ad esempio vizio di motivazione); o, se la legge tace, riesce semplicemente irregolare, ferma restando la sua piena validità. In altri termini, la violazione di un dovere non è incompatibile con l'esercizio di un potere.

\section{REGOLE DI INCLUSIONE E DI ESCLUSIONE PROBATORIA}

Le regole sulla ammissibilità/inammissibilità della prova altrimenti dette, regole di inclusione o di esclusione probatoria - sono di stretta competenza del legislatore, a differenza, come si dirà, dei criteri di valutazione della prova: il giudice deve limitarsi a renderle operative, non essendo pensabile che egli possa, di volta in volta, individuare e variare i criteri di ammissione delle prove.

3 Con la prova inammissibile non va confusa la prova di cui il giudice non ammetta l'assunzione in quanto manifestamente irrilevante o superflua; qui la prova non è inammissibile nel senso proprio della parola, ma semplicemente ritenuta inutile, allo stato degli atti, dal giudice; il che, peraltro, non esclude una sua successiva assunzione, quando se ne manifesti l'utilità.

4 F. Cordero, Tre studi sulle prove penali, Giuffrè, Milano, 1963, 61 s.; ID, Le situazioni soggettive nel processo penale, Giappichelli, Torino, 1957, passim; ID, Procedura penale, 9 ed., Giuffrè, Milano, 2012, 610 s. 
In particolare, le regole di esclusione possono essere 'assolute' (come per gli scritti anonimi, per le dichiarazioni estorte, per le voci correnti nel pubblico, ecc.), ossia incondizionatamente operanti; o 'relative', quando intervengono solo in rapporto ad una determinata proposizione da provare o in una determinata fase (ad esempio, le dichiarazioni unilateralmente raccolte dagli organi inquirenti non costituiscono prova in dibattimento sul tema della colpevolezza, ma sono pienamente utilizzabili ad altri fini, come nei provvedimenti cautelari o nei riti negoziali).

Da notare che talvolta la regola di esclusione probatoria sembra cadere non sulla prova come premessa probatoria, ma sulla proposizione da provare: ad esempio, quando l'art. 194 c.p.p. afferma che il testimone non può deporre sulla moralità dell'imputato o sulle voci correnti nel pubblico, il divieto non riguarda la prova in sé (la testimonianza), ma l'oggetto, il tema su cui verte (la 'moralità' o le 'voci correnti'). Ma, a ben vedere, se la legge sottrae alla prova un certo tema è solo al fine di evitare che quello stesso tema si converta, nella sequenza argomentativa, in una nuova premessa probatoria ${ }^{5}$, vale a dire in prova rispetto alla proposizione finale, rappresentata dalla colpevolezza; in altri termini, si vieta la testimonianza sulla moralità dell'imputato o sulle voci correnti nel pubblico affinché né l'una né le altre assumano valore probatorio per la ricostruzione del fatto. Dunque, la regola di esclusione coinvolge sempre le premesse probatorie.

\section{Criteri di valutazione}

Ben diversa la struttura dei criteri di valutazione che, a differenza delle regole di esclusione, implicano prove validamente costituite e riguardano il passaggio da queste alla proposizione da provare. In linea di massima - e soprattutto nell'ambito del modello accusatorio - l'organo competente a definire i criteri di valutazione è il giudice stesso, proprio perché coinvolgono il maggiore o minore valore che, nel caso concreto, una prova può assumere rispetto alla proposizione da provare.

5 Sulle ‘sequenze probatorie', v. P. Ferrua, La prova nel processo penale, cit., $76 \mathrm{~s}$. 
Questo non esclude che talvolta il legislatore ritenga opportuno fissare determinati criteri di valutazione, come accade, nel nostro ordinamento, con l'art. 192 commi 2 e 3 c.p.p., relativi agli indizi e alle dichiarazioni rese dal coimputato. Essendo i criteri per lo più enunciati nella forma ' $q$ vale come prova di $p$ solo in presenza di $r$ ' (così l'art. 192 c.p.p.), si può essere tentati di convertirli in una regola di esclusione del tipo ' $q$, in assenza di $r$ ', è un dato inutilizzabile'.

Ad esempio, dire che le dichiarazioni del coimputato sono valutate unitamente agli altri elementi che ne confermano l'attendibilità - così il criterio fissato dall'art. 192 comma 3 c.p.p. ${ }^{6}$ - non equivale a dire che, mancando tali elementi, la dichiarazione è inutilizzabile? È istintivo rispondere positivamente, ma è un errore, favorito ancora una volta dall'ambiguità del termine 'prova'. Il criterio legale non incide sulla valida costituzione della prova, intesa come dato valutabile dal giudice; influisce solo sul valore della prova sino ad annullarlo in assenza di certi requisiti. Nell'ipotesi di cui sopra, la dichiarazione del coimputato è 'prova' (si allude ad una potenzialità del dato legittimamente acquisito) e, come tale, dev'essere valutata dal giudice; ma, in forza della regola legale, è inidonea a 'provare' il fatto dichiarato, in assenza di riscontri.

Talvolta il criterio di valutazione si avvicina molto alla regola di esclusione probatoria. È il caso del criterio contenuto nella seconda parte dell'art. 111 comma 4 Cost. e nell'art. 526 comma 1-bis c.p.p.: «la colpevolezza non può essere provata sulla base delle dichiarazioni rese da chi, per libera scelta, si è sempre volontariamente sottratto all'esame da parte dell'imputato o del suo difensore». A prima vista appare come una regola di esclusione perché le dichiarazioni in parola non possono in alcun modo provare la colpevolezza, neppure se unite ad altre prove o utilizzate come semplici elementi di riscontro. In realtà, da un'attenta lettura della norma si evince che è, invece, possibile il loro uso in chiave difensiva, a favore dell'imputato; si tratta, quindi, di un criterio di valutazione grazie al quale le dichiarazioni potranno essere acquisite al processo e valutate in utilibus, ferma restando la loro assoluta inidoneità a provare la colpevolezza.

6 Quanto all'art 192 comma 2 c.p.p. v. infra par. 5 
Insomma, una cosa è che un dato non possa essere valutato perché inutilizzabile (id est, non acquisibile come prova), quindi sottratto a priori al convincimento giudiziale; un'altra che, per effetto dei criteri legali, il valore di una prova si riduca o addirittura scenda a zero rispetto a determinati esiti: la negazione che nel primo caso preclude la valutazione, nel secondo ne orienta o ne condiziona negativamente l'esito.

\section{AMMISSIBILITÀ ED EFFICACIA PERSUASIVA DELLA PROVA}

Le questioni relative all'ammissibilità della prova sono, dunque, ben distinte da quelle relative all'efficacia persuasiva che in concreto può esercitare il singolo dato. Purtroppo, nei discorsi sulla prova si registra spesso una confusione tra i due piani.

Analizziamo, ad esempio, l'enunciato ' $x$ non è una prova'. Correttamente inteso, significa che $x$ non costituisce una valida premessa probatoria, e quindi non è valutabile dal giudice sul tema $y$ (ad esempio, perché raccolto senza l'osservanza delle regole del contraddittorio o illegittimamente acquisito al processo). Ma, nel senso improprio in cui talora è usato nel linguaggio corrente, sta a significare che $x$, pur costituendo una valida premessa, si rivela, nel caso concreto, inidoneo a 'provare' $y$ (ad esempio, una testimonianza ritenuta inattendibile o irrilevante). C'è una differenza abissale tra dire che ' $x$ è (o non è) prova sul tema $y$ ' e dire che ' $x$ prova o non prova $y$ ': nel primo senso si afferma o si nega una potenzialità probatoria; nel secondo, si afferma o si nega l'esito positivo della prova. La prova su y, ossia la premessa probatoria, non va confusa con la prova di y, ossia con il risultato probatorio, nonostante sia assai frequente la sovrapposizione dei due concetti.

Dovrebbe essere chiaro, infatti, che una testimonianza non cessa di essere 'prova' per il solo fatto che sia vaga, irrilevante o persino falsa; così come costituisce una prova sul tema della colpevolezza la circostanza che l'imputato fosse in buoni o cattivi rapporti con la vittima, ne fosse o no geloso, pur trattandosi di elementi di per sé del tutto inidonei a provare il gesto omicida. 'Prova' è tutto ciò che sia legittimamente acquisito al processo e, quindi, valutabile dal giudice in ordine ad un qualsiasi tema probatorio; e poco importa, ai fini del concetto di prova, che un dato 
valutabile si riveli in concreto più o meno persuasivo, più o meno rilevante rispetto alla proposizione da provare.

È netta la differenza tra una prova vietata dalla legge, quindi non acquisibile al processo, e una prova manifestamente irrilevante o sovrabbondante. A norma dell'art. 190 c.p.p. il giudice esclude «le prove vietate dalla legge e quelle che manifestamente sono superflue o irrilevanti». Il tenore della disposizione può indurre a ritenere che tanto le prove vietate dalla legge quanto quelle manifestamente irrilevanti o superflue siano inammissibili, dovendo il giudice escluderle. Ma non è così. Inammissibile nel significato proprio della parola è la prova vietata dalla legge, ossia oggetto di una regola di esclusione probatoria (ad esempio, gli scritti anonimi, la testimonianza dell'imputato, i mezzi che ledono la libertà di autodeterminazione, ecc.); vale a dire, la prova che il giudice non ha il potere di assumere. Quella che il giudice esclude perché manifestamente irrilevante o superflua non è inammissibile nel senso appena specificato; né sarebbe corretto dire che il giudice sia privo del potere di assumerla. Viene (provvisoriamente) esclusa perché ritenuta, allo stato, inutile a fini decisori.

Il giudizio sulla superfluità e irrilevanza, infatti, non riguarda la prova in sé, intesa come premessa probatoria, ma il suo rapporto con il contesto in cui si colloca, e, più specificamente, con la proposizione da provare (nel caso della irrilevanza) o con le altre prove (nel caso della superfluità). È un giudizio che, in qualche misura, attiene al merito e la cui anticipazione in sede preliminare, ossia di ammissione della prova, si giustifica per esigenze di ragionevole durata del processo; sarebbe una perdita di tempo assumere prove palesemente superflue o irrilevanti. Ma, proprio per il rischio di sconfinamento nel merito e di perdita di imparzialità del giudice, la legge circoscrive l'estensione delle due tipologie con l'avverbio 'manifestamente'. Si spiega così che il provvedimento di esclusione della prova manifestamente superflua o irrilevante sia pronunciato 'allo stato degli atti', essendo sempre rivedibile al variare del quadro probatorio. La prova già esclusa può, al mutare del rapporto con le altre prove o in una diversa prospettiva di ricostruzione del fatto, rivelarsi utile e pertinente; per converso, una prova vietata dalla legge resta sempre inammissibile, quale che sia il contesto in cui si inserisce. 


\section{IL CONCETTO DI PROVA INDIZIARIA}

Ovunque vi sia una proposizione da provare, necessariamente devono esservi prove, non essendo possibile provare se non attraverso prove. L'idea che qualcosa possa essere 'provato' da entità diverse dalle prove - comunque le si chiami, elementi, indizi, ecc. - è una contraddizione in termini; se $x$ è provato da $y$ (solo o congiunto ad altre prove), $y$ non può che essere una prova. Per converso, è altrettanto evidente che non tutte le 'prove' sortiscono necessariamente l'effetto di 'provare': vi sono prove, ossia dati valutabili dal giudice, che in concreto, a causa della loro irrilevanza o intrinseca debolezza, risultano ininfluenti sulla proposizione da provare o addirittura ne costituiscono la prova negativa.

Assai controversa è la categoria delle 'prove indiziarie'. A nostro avviso, occorre distinguere tra la nozione che affiora dall'art. 192 c.p.p., costruito sul malfermo presupposto di una intrinseca debolezza degli 'indizi', e il concetto teoretico di prova critico-indiziaria, ben delimitato nei suoi contorni; concetto che, nella prospettiva di Francesco Carnelutti, poi perfezionata da Franco Cordero, si contrappone a quello di 'prova dichiarativa'.

L'art. 192 c.p.p. è una disposizione malriuscita, che risente della logica inquisitoria, incline a stabilire astratte gerarchie tra le diverse tipologie di prova ${ }^{7}$. Nel modello accusatorio il valore delle prove va misurato nel contesto del singolo processo, dove l'unità di rilevanza empirica è rappresentata dall'intero quadro probatorio.

Nel tentativo di dare un senso all'art. 192 c.p.p., alcuni autori identificano gli indizi con le prove basate su massime di esperienza anziché su leggi scientifiche, in altri termini con prove fondate sulla doxa anziché sull'episteme ${ }^{8}$. Ma, impostata in questi termini, la distinzione,

7 Cordero definisce l'art. 192 comma 2 c.p.p. «un tentativo velleitario d'imporre regole alla clinica giudiziaria», una «inutile» disposizione destinata ad alimentare «confusione» (Procedura, cit., 626, 623).

8 In tal senso, v., fra gli altri, A. Nappi, Guida al codice di procedura penale, 9 ed., Giuffrè, Milano, 2004, 210; G. Ubertis, Fatto e valore nel sistema probatorio penale, Giuffrè, Milano, 1979, 112 s.; ID., Documenti e oralità nel nuovo processo penale, in Studi in onore di G. Vassalli, II, Giuffrè, Milano, 1991, 304 s., e, più di recente, del medesimo autore, Processo indiziario e valutazione probatoria, in Diritto\&Questioni pubbliche, 2020/1, giugno, 317 s., dove si afferma 
pur riflettendo il preconcetto che traspare dall'art. 192 c.p.p., risulta incerta, posto che nell'ambito del settore induttivo è impossibile distinguere con un taglio netto le inferenze basate sulla scienza da quelle fondate sulle massime di esperienza; ad esempio, la psicologia è senza dubbio una scienza, ma le inferenze sul comportamento umano sono ben lungi dall'avere la solidità delle scienze così dette dure come la fisica o la chimica. La scienza, ovvero l'episteme sfuma nell'esperienza corrente e nella doxa senza una netta soluzione di qualità'.

Tanto meno si potrebbe contrapporre la prova indiziaria alle prove dalle quali la proposizione da provare discenderebbe per 'necessità logica'. Nel processo, dove si riscostruiscono fatti del passato, nessuna prova è in grado di provare in modo 'indubitabile', vale a dire nel senso propriamente deduttivo, tipico delle scienze formali; il massimo, ma al tempo stesso il minimo, che si può pretendere per provare una qualsiasi proposizione, è la prova oltre ogni ragionevole dubbio, dove l'aggettivo 'ragionevole' tempera e riduce la pretesa di assoluta certezza. Fuori dal settore deduttivo - dove la prova assume la forma della dimostrazione, come tale indubitabile - l'oltre ogni ragionevole dubbio è il solo standard compatibile con il concetto di 'provare'; non è possibile superare quel livello, ma scendere al di sotto significherebbe sconfinare nella zona delle ipotesi, delle illazioni e, per dir tutto, dei sospetti ${ }^{10}$.

che «un processo può denominarsi indiziario (in sostanziale corrispondenza con il già rammentato uso comune della terminologia) quando la conclusione inferenziale della sequenza probatoria si basa non su prove in senso stretto (rappresentative o critiche), ma su indizi (evitando di adoperare l'ambigua e fuorviante espressione 'prove indiziarie'), caratterizzandosi quindi per la modalità logica non della necessità, ma della semplice possibilità». Perché definire 'ambigua' e 'fuorviante' l'espressione 'prove indiziarie'? Se gli indizi non fossero prove, non potrebbero provare un bel nulla, neppure se gravi, precisi e concordanti.

9 Nella stessa testimonianza, anche quella sul fatto principale, l'inferenza, che dalla dichiarazione consente di passare al fatto dichiarato, non si può certo definire fondata 'scientificamente'; e, da un certo punto di vista, se c'è una prova debole, è proprio quella dichiarativa.

10 A mutare non è mai lo standard probatorio, che resta sempre contrassegnato dalla regola dell'oltre ogni ragionevole dubbio, ma la proposizione da provare. Mentre ai fini della condanna la proposizione da provare è la 'colpevolezza', ai fini dei provvedimenti cautelari le proposizioni da provare sono i pericula libertatis e il fumus boni iuris rappresentato dai gravi indizi di colpevolezza, 
Altri autori identificano la prova indiziaria con la prova 'indiretta', ossia con una prova che ha ad oggetto un fatto secondario, dal quale poi si risale al fatto principale ${ }^{11}$. Ma in tal modo escono confuse tipologie distinte. Mentre la prova indiziaria è un'entità singola, la prova indiretta nasce dalla congiunzione di due prove disposte in sequenza, l'una rispetto all'altra; la proposizione che viene provata dalla prima prova diventa la prova di una nuova proposizione con un meccanismo che potenzialmente potrebbe andare avanti all'infinito.

Se un teste dichiara di avere visto l'imputato aggirarsi nel luogo del delitto e si utilizza questa dichiarazione per provare la responsabilità dell'imputato, le prove sono due. La prima è rappresentata dalla testimonianza (prova dichiarativa) che prova la presenza dell'imputato nel luogo del delitto; la seconda da questa presenza, la quale, a sua volta, funge da prova (critico-indiziaria) rispetto alla commissione del fatto da parte dell'imputato: la proposizione provata nella prima inferenza si converte in proposizione probatoria per una seconda inferenza. Altrettanto accade nella c.d. testimonianza indiretta che, in realtà, è costituita da due prove dichiarative poste in sequenza, quella del teste diretto e quella del teste indiretto che riferisce quanto appreso.

L'unica distinzione, che riesce a delimitare con precisione la categoria delle prove critico-indiziarie, è quella che le contrappone alle prove dichiarative; tutto ciò che non è prova dichiarativa appartiene al genus delle prove critico-indiziarie che risultano così individuate per

vale a dire dalla 'probabile colpevolezza'. Sul ragionevole dubbio come unico standard probatorio, v. M.L. Busetto, Il contraddittorio inquinato, Cedam, Padova, 2009, 173; P. Ferrua, Onere della prova, regola di giudizio e standard probatorio: alla ricerca della perduta proposizione da provare, in Cass. pen., 2020, 2639 s.; P. Paulesu, La presunzione di non colpevolezza dell'imputato, 2 ed., Giappichelli, Torino, 2009, 228 s. Contra, per la sussistenza di vari standard probatori, v. F. M. Iacoviello, La Cassazione penale. Fatto, diritto e motivazione, Giuffrè, Milano, 2013, p. 432, 632 s.; F. Caprioli, voce Condanna (dir. proc. pen.), in Enc. dir., Annali, vol. II, t. 1, Giuffrè, Milano, 2008, 111 s.; C. Santoriello, Il vizio di motivazione tra esame di legittimità e giudizio di fatto, Utet, Torino, 2008, $58 \mathrm{~s}$.

11 In questa prospettiva v., ad esempio, G. Lozzi, Lezioni di procedura penale, 8 ed., Giappichelli, Torino, 2013, 210; M. Scaparone, Procedura penale, vol. I, 2 ed., Giappichelli, Torino, 2010, 301 s.; D. Siracusano - A. Galati - G. Tranchina - E. Zappalà, Diritto processuale penale, Giuffrè, Milano, 2018, 280. 
differenza o sottrazione dalla prova dichiarativa. È assai difficile definire la prova critico-indiziaria sulla base di una sua nota peculiare (come si è visto, le definizioni correnti o soffrono di nebulosità ai confini o confondono la prova indiziaria con quella indiretta); conviene, dunque, definirla per esclusione rispetto alla prova dichiarativa, i cui connotati sono invece facilmente identificabili.

La prova dichiarativa contiene, già espressa al suo interno, la proposizione di cui si rende garante, ossia la proposizione da provare: 'Tizio afferma che $x$ ' è la proposizione o premessa probatoria, ' $x$ ' è la proposizione da provare. All'osservatore resta solo da stabilire se ' $x$ ' sia vero o falso, essendo già individuato il tema della prova. La prova critico-indiziaria è, per così dire, 'muta', nel senso che tocca all'interprete accertare quale proposizione essa possa provare; la qualifica 'criticoindiziaria' sta, appunto, ad indicare questa attività di decifrazione del tema oggetto della prova.

Così impostata, la distinzione non ha nulla a che vedere con la maggiore o minore affidabilità dell'uno o dell'altro tipo di prova: la professione di innocenza dell'imputato è una prova dichiarativa, ma non vale certo più della sua impronta digitale sull'arma del delitto o della fotografia che lo coglie nell'atto di commettere il delitto. L'efficacia persuasiva delle prove va accertata in concreto, nel singolo processo e alla luce dell'intera evidenza disponibile; è illusorio pensare di definirla in astratto, isolando questa o quella tipologia di prova, come accadeva nel processo inquisitorio; categorie del genere riescono inutili, quando non sono dannose.

La definizione di Carnelutti ${ }^{12}$, che contrapponeva le prove 'rappresentative' (o storiche) a quelle critico-indiziarie, aveva l'effetto di includere nelle prime anche la fotografia e la videoregistrazione che certamente riproducono e rappresentano fatti del passato ${ }^{13}$; tuttavia,

12 F. Carnelutti, Lezioni sul processo penale, vol. I, Edizioni dell'Ateneo, Roma, 1946,213 s.; ID., La prova civile [1015], ristampa, Giuffrè, Milano, 1992, 92 s.; ID., Principi del processo penale, Morano, Napoli, 1960, 162 s.; ID., Diritto e processo, Morano, Napoli, 1958, 129 s.

13 Roland Barthes identifica il noema della fotografia in «È stato» (La camera chiara. Note sulla fotografia, Einaudi, Torino, 1980, 78). Anche l'impronta digitale rientra nella categoria carneluttiana delle prove rappresentative. 
sono prove critico-indiziarie, dato che la proposizione da provare (la realtà della cosa fotografata o della scena registrata) non è 'espressa' ma indotta dall'osservatore sulla base di leggi ottiche ed acustiche. Si aggiunga che, propriamente parlando, il linguaggio non 'rappresenta' nulla, ma si limita a trasmettere significati. Opportunamente Cordero riduce la classe carneluttiana delle prove rappresentative o storiche alle sole prove dichiarative o funzioni narrative ${ }^{14}$.

Resta da determinare quando una prova possa definirsi dichiarativa. I connotati, necessari e sufficienti, sono due. Il primo è un atto comunicativo, ossia un atto volto intenzionalmente a trasmettere significati: il che avviene, per lo più, attraverso il linguaggio, ossia parlando; ma può realizzarsi anche con gesti, quando questi funzionano alla stregua di un linguaggio, ossia sono codificati come equivalenti di parole (così accade nel dialogo tra sordomuti).

Mentre l'uso del linguaggio rappresenta già di per sé un atto comunicativo, salvo situazioni eccezionali (come le parole pronunciate in stato di incoscienza), non è facile stabilire in concreto quando un comportamento non verbale costituisca un atto comunicativo; l'indagine nel nostro ordinamento assume rilievo, ad esempio, al fine di disporre una videoregistrazione nel domicilio privato, ammissibile secondo la giurisprudenza solo per i comportamenti comunicativi, rispetto ai quali è equiparabile ad un'intercettazione. Il criterio di demarcazione, chiaro a livello teorico, ma di non semplice applicazione pratica, può essere così sintetizzato: un comportamento è comunicativo quando l'emittente si propone di trasmettere un'informazione, attraverso il riconoscimento della sua intenzione di trasmetterla ${ }^{15}$. Come si nota, il criterio s'incentra sulle intenzioni, non sempre agevolmente decifrabili,

14 F. Cordero, Procedura [2012], cit., $581 \mathrm{s.}$

15 Cfr. P. Grice, Logica e conversazione (1989), trad. it., Il Mulino, Bologna, 1993, 58 secondo cui «S ha voluto dire qualcosa con $x$ quando $S$ ha avuto l'intenzione che $x$ producesse un certo effetto sull'ipotetico destinatario attraverso il riconoscimento di questa intenzione»; ma v. anche L. J. Prieto, Saggi di semiotica, II, Sull'arte e sul soggetto, Pratiche, Parma, 1991, 104; D. Sperber-D. Wilson, La pertinenza (1986), trad. it., Anabasi, Milano, 1993. Per lo sviluppo del tema nel processo penale, v. P. Ferrua, La prova nel processo penale, cit., $68 \mathrm{~s}$. 
dell'emittente, indipendentemente dalla circostanza, di per sé irrilevante, che l'informazione sia recepita dal destinatario ${ }^{16}$.

Il secondo connotato è il carattere 'apofantico' della dichiarazione, ossia la circostanza che l'enunciato sia vero o falso. Se l'enunciato non è né vero né falso - come gli ordini, i consigli, le preghiere ecc. - la prova non è dichiarativa, ma critico- indiziaria; infatti, la proposizione, oggetto di prova, non è contenuta nell'enunciato stesso, ma va, di volta in volta, individuata. Mi spiego con un esempio. Se qualcuno ha detto 'la porta è aperta', si è in presenza di una prova dichiarativa nella quale viene enunciata la proposizione di cui vuole rendersi garante. Ma, se ha detto 'apri la porta', si è in presenza di una prova critico-indiziaria, nella quale occorre individuare la proposizione di cui può essere la prova ( $\mathrm{ad}$ esempio, che la porta era chiusa, che l'emittente intendeva avviare un dialogo riservato con l'interlocutore, il rapporto confidenziale segnalato dal verbo in seconda persona, ecc. $)^{17}$.

${ }^{16}$ Chiarisco il concetto con un esempio, immaginando che Tizio, seduto al tavolo di un ristorante, agiti il bicchiere vuoto. Possiamo distinguere le seguenti situazioni. Nella prima il soggetto, utilizzando il gesto come un linguaggio, intende apertamente trasmettere al personale l'invito a servirgli da bere; in tal caso si realizzano tutte le componenti della comunicazione, anche se, come vedremo, questa non è di tipo propriamente dichiarativo, trattandosi di un ordine che in sé non è né vero né falso.

Nella seconda Tizio, senza palesare le sue intenzioni, vuole indurre il personale a servirgli da bere, con il proposito che il suo gesto non sia recepito come una richiesta, ma sia decifrato come un segno naturale (quindi, critico-indiziario) di sete: qui l'informazione viene intenzionalmente trasmessa, ma, a differenza del primo caso, il gesto non realizza un atto comunicativo, perché manca la volontà che sia riconosciuto l'intento informativo.

Nella terza, infine, il soggetto agita il bicchiere perché assetato o, semplicemente, perché nervoso, ma senza alcuna intenzione informativa: il gesto può senza dubbio trasmettere varie informazioni in chiave critico-indiziaria, ma risulta slegato da qualsiasi proposito informativo. È evidente che solo nel primo caso il gesto funziona propriamente come un linguaggio, ossia trasmette significati nel senso non naturalistico della parola.

17 Naturalmente, nel processo, che qualcuno abbia detto 'apri la porta' risulterà o dall'affermazione di un testimone o da una registrazione. In entrambi i casi si è in presenza di due prove disposte in sequenza. Nel primo caso, una prova dichiarativa (la testimonianza) su una prova critico-indiziaria ('ordine di aprire la porta); nel secondo, una prova critico-indiziaria (la registrazione) su una prova critico-indiziaria (l'ordine impartito). 


\section{Prove atipiche}

Si discuteva, vigente il codice abrogato, se l'elencazione legislativa delle prove dovesse considerarsi tassativa. Il progetto del codice di procedura penale del 1978 era orientato in senso affermativo, ma nel codice vigente è prevalsa l'opposta soluzione. L'art. 189 c.p.p. dispone che «quando è richiesta una prova non disciplinata dalla legge, il giudice può assumerla se essa risulta idonea all'accertamento dei fatti e non pregiudica la libertà morale della persona. Il giudice provvede all'ammissione, sentite le parti sulle modalità di assunzione della prova».

La disposizione è palesemente diretta a consentire l'ingresso nel processo dei nuovi mezzi cognitivi resi disponibili dall'evoluzione scientifica e tecnologica (si pensi allo sviluppo delle prove neuroscientifiche). La verifica dell'idoneità all'accertamento dei fatti serve ad escludere ogni strumento che appaia gnoseologicamente inadeguato, perché non ancora sperimentato a sufficienza o, all'opposto, perché caduto in discredito nella comunità degli esperti. Trattandosi di mezzi, le cui modalità di assunzione non sono definite dalla legge, è corretto che, prima di disporli, il giudice individui tempi e modi di formazione della prova, ascoltando il parere delle parti.

Non è chiaro se l'ammissione delle prove neuroscientifiche nel processo debba svolgersi secondo le regole ordinarie fissate dall'art. 190 c.p.p. o secondo quelle contemplate dalla disposizione in esame. Quanto al controllo sull'idoneità all'accertamento dei fatti e sulla mancanza di pregiudizio per la libertà morale, non vi è differenza tra l'una e l'altra soluzione: la scientificità del metodo - in nome della quale sono messe al bando tecniche non accreditate dal punto di vista epistemologico - è implicita nel concetto stesso di perizia (o di consulenza tecnica); e, a sua volta, la libertà di autodeterminazione è incondizionatamente protetta dall'art. 188 c.p.p. ${ }^{18}$.

La differenza sta nella regola di ammissione: per le prove 'atipiche' l'art. 189 c.p.p. impegna al contraddittorio anticipato, dovendo il giudice

18 Cfr. in senso critico rispetto all'utilizzo dell'art. 189 c.p.p. per introdurre nel processo penale la c.d. 'prova scientifica', V. Bozio, La prova atipica, in P. Ferrua-E. Marzaduri-G. Spangher (a cura di), La prova penale, Giappichelli, Torino, 2013, $57 \mathrm{~s}$. 
sentire le parti sulle modalità della loro assunzione. Può essere questa una buona ragione per considerare 'atipiche' le prove neuroscientifiche; ma, dal punto di vista esegetico, la soluzione incontra qualche ostacolo nella formulazione dell'art. 189 c.p.p., dove si parla di 'prova non disciplinata' dalla legge, mentre le prove in esame entrano nel processo attraverso la perizia o la consulenza tecnica che sono legislativamente regolate. Il problema, in sostanza, è che l'art. 189 c.p.p. riferisce l'atipicità all'assenza di una previsione legislativa per l'ingresso della prova nel processo, mentre nel caso delle neuroscienze l'atipicità sta nella novità del metodo, ancora in fase sperimentale. Una possibile, ragionevole soluzione può essere rappresentata da un'applicazione analogica dell'art. 189 c.p.p., tale da includervi ogni nuova tecnica di indagine, ritenuta idonea all'accertamento dei fatti, quale che sia il veicolo di ingresso nel processo penale (perizia, esperimento giudiziale o altro mezzo).

\section{LIBERTÀ DI AUTODETERMINAZIONE}

Ai sensi dell'art. 188 c.p.p. «non possono essere utilizzati, neppure con il consenso della persona interessata, metodi o tecniche idonei a influire sulla libertà di autodeterminazione o ad alterare la capacità di ricordare e di valutare i fatti» ${ }^{19}$. La disposizione riguarda anzitutto gli atti in cui la persona (imputato o testimone) interviene come soggetto parlante, quindi le prove di tipo dichiarativo. L'atto di parola è valido in quanto sia frutto di libera volizione; una testimonianza resa in stato di coazione psichica è una pseudo-testimonianza, priva di qualsiasi rilevanza probatoria. Non sono pertanto ammissibili la narcoanalisi o l'ipnosi ${ }^{20}$ né

19 Sulla libertà di autodeterminazione nel processo penale v. L. SCOMPARIN, La tutela del testimone nel processo penale, Cedam, Padova, 2001, $101 \mathrm{~s}$.

20 Per l'inammissibilità dell'ipnosi v. Corte di Assise di Caltanisetta 28 aprile 1999, in Foro it., 2000, II, 248 (L'attività del perito non è sottratta alla regola generale di cui all'art. 188 c.p.p., la quale si riferisce a tutte le forme di assunzione della prova; ne consegue che sono inutilizzabili i risultati dell'accertamento peritale, avente ad oggetto l'attitudine a testimoniare di individuo minore di età, condotto attraverso al sottoposizione ad ipnosi del minore medesimo allo scopo di accertare la presenza e la natura di elementi condizionanti la sua psiche). Per la responsabilità disciplinare di un pubblico ministero che aveva disposto una consulenza tecnica tramite seduta ipnotica allo 
altre tecniche manipolative che tolgano al soggetto il controllo su ciò che intende dire o semplicemente lo affievoliscano, impedendogli di esprimere liberamente il proprio pensiero (come gli interrogatori di 'terzo grado', le minacce, le promesse di favori, le domande nocive ecc.).

Per analoghe ragioni è inammissibile una costrizione del soggetto ad atti che richiedano un comportamento collaborativo, un facere. Il divieto non coinvolge, invece, gli atti in cui, per la loro stessa natura, la persona intervenga come corpo, come oggetto di ispezione, di prelievo o di riconoscimento; atti che implicano un atteggiamento passivo di chi vi è sottoposto, che si sostanziano in un pàtere. In questi casi l'esecuzione coatta dell'atto non è a priori inammissibile, purché sia prevista e regolata dalla legge come richiede l'art. 13 comma 2 Cost., secondo cui «non è ammessa forma alcuna di detenzione, di ispezione o perquisizione personale, né qualsiasi altra restrizione della libertà personale, se non per atto motivato dell'autorità giudiziaria e nei soli casi e modi previsti dalla legge» ${ }^{21}$.

\section{Memory detection}

Si discute se sia compatibile con i principi del nostro ordinamento la memory detection che è stata messa a punto in Italia dal neuroscienziato Giovanni Sartori e che ha trovato applicazione in alcuni processi (il c.d. IAT). È una tecnica, costruita sui tempi di reazione alle domande, che consiste all'incirca nel chiedere al soggetto di classificare in modo rapido e accurato come vere o false le frasi che compaiono nel monitor di un computer; lo IAT si basa sulla teoria, di indubbio fondamento, che un ricordo genuino ha rapidi tempi di reazione, mentre la sua falsificazione determina il loro aumento, imputabile al conflitto cognitivo che il soggetto deve superare per fornire una risposta non conforme al ricordo naturale ${ }^{22}$.

scopo di ravvivare la memoria del testimone, Sez. un. civ. 1 febbraio 2008, in Cass. pen., 2008, 3617.

21 In questi termini si è espressa, in rapporto alla prova del DNA, la Corte costituzionale con sentenza n. 238 del 1996.

22 Una versione primordiale e divertente dello IAT è descritta dal filosofo Bertrand Russell: «Come ognuno sa, l'associazione offre un metodo per prendere in trappola i criminali. Voi state interrogando, mettiamo, un uomo che sospettate abbia tagliato la gola alla moglie con un coltello. Voi dite una parola, 
Numerosi autori rispondono negativamente, per il fatto stesso che queste tecniche si risolvano in varie forme di introspezione mentale ${ }^{23}$; ed è per tale ragione che nel nostro processo non ha mai trovato ingresso il poligrafo o lie detector. Tuttavia, non è priva di fondamento l'osservazione di Gian Franco Ricci secondo cui la libertà di autodeterminazione non è pregiudicata né dal lie detector, che si limita a registrare la variazione di certi parametri fisiologici, né da altri metodi volti a scoprire la menzogna attraverso l'analisi delle reazioni a certe domande ${ }^{24}$.

In effetti, prendendo ad esempio lo IAT, il soggetto si sottopone al test per libera scelta; e, a differenza di quanto accadrebbe con la narcoanalisi o con l'ipnosi, resta pienamente libero di definire vere o false le frasi che gli vengono proposte. Certo, sa che, mentendo, con ogni probabilità sarà scoperto, ma non ci sentiremmo di teorizzare un diritto a mentire senza essere scoperti in base alle modalità extralinguistiche delle risposte. Nessuno nega che l'analisi dei tratti prosodici del discorso (esitazioni, tono della voce, rossore del volto, movimenti del corpo, esitazioni nelle risposte ecc.) fornisca preziosi indici per valutare la credibilità del dichiarante; e qui, in definitiva, è proprio tramite l'analisi dei tratti paralinguistici (i tempi di reazione alle domande) che si decifrano le menzogne.

Le ragioni di perplessità verso le tecniche in esame, a mio avviso, più che nella lesione della libertà del volere, forse stanno altrove; $\mathrm{o}$ meglio, solo indirettamente si collegano al tema dell'autodeterminazione. Derivano dalla struttura stessa di questi mezzi nei quali la persona, proprio

ed egli deve rispondere con la prima parola che gli viene in mente. Voi dite 'gatto' ed egli risponde 'cane'; voi dite 'politico' ed egli dice 'ladro'; voi dite 'coltello' ed egli ha un primo impulso a dire 'gola', ma sa che è meglio non dirlo, così, dopo lunga esitazione dice 'forchetta'. La durata dell'esitazione mostra la sua resistenza» (B. Russell, Storia delle idee nel XIX secolo, Mondadori, Milano, 1969, 139 s.).

23 V., fra i molti, F. Cordero, Procedura [2012], cit., 616; G. Di Chiara, Il canto delle sirene: Processo penale e modernità scientifico-tecnologica: prova dichiarativa e diagnostica della verità, in Criminalia, 2007, 19 s.; V. Grevi, Prove, in G. Conso-V. Grevi-M. Bargis, Compendio di procedura penale, 6 ed., Cedam, Padova, 313 s.; F. Grifantini, sub art. 188, in Commentario breve al codice di procedura penale, a cura di G. Conso-V. Grevi, Cedam, Padova, 2005, 530. 
nell'atto di parola che dovrebbe vederla come partecipe di un processo comunicativo, degrada a mero oggetto di osservazione e di analisi ${ }^{25}$. L'aspetto vagamente inquietante è che qui l'atto di parola non venga più in rilievo come momento di dialogo e occasione di ascolto, ma sia analizzato e per così dire trattato 'chimicamente' allo scopo di estrarne informazioni alla stessa stregua con cui si effettua un esame ematologico o si ispeziona un organo.

Anche nella valutazione della testimonianza si tiene conto dei tratti paralinguistici del discorso; ma lì assumono rilevanza come elementi di riscontro ad un dialogo che si svolge nel contraddittorio e in cui si parla per essere creduti. Qui, invece, il rapporto si inverte perché non si risponde per essere ascoltati né per essere creduti; la parola non è più un mezzo comunicativo, ma serve da elemento di informazione solo attraverso l'analisi dei tempi di reazione (o, in altre tecniche, delle neuroimmagini). Nell'inevitabile bilancio tra costi e benefici, forse l'antico e illustre metodo della cross-examination resta ancora il metodo migliore per il controllo sulla credibilità del testimone.

\section{DiVIETI PROBATORI}

Divieti probatori e regole di esclusione obbediscono a molteplici esigenze. Spesso il fine perseguito è il corretto accertamento dei fatti, talvolta il diritto di difesa o altri diritti individuali (come la tutela della segretezza delle comunicazioni, nei casi in cui è vietata l'intercettazione) o, ancora, una pluralità di valori. Ad esempio, la regola che nega valore probatorio alle dichiarazioni raccolte fuori dal contraddittorio non corrisponde soltanto ad un elementare esigenza difensiva, ma assume anche un valore epistemico, essenziale per la solida ricostruzione dei fatti. Contrariamente a quanto alcuni asseriscono, il contraddittorio non è nemico, ma alleato nella ricerca della verità: le sentenze con cui la Corte costituzionale ha liquidato le regole di esclusione probatoria dettate a tutela del contraddittorio appartengono alle pagine più desolanti della sua giurisprudenza ${ }^{26}$.

25 Cfr. P. Ferrua, La prova, cit., 318 s.

26 Sentenze nn. 24, 254 e 255 del 1992.

Rev. Bras. de Direito Processual Penal, Porto Alegre, v. 7, n. 1, p. 215-246, jan.-abr. 2021. 
Il divieto probatorio è fissato dalle disposizioni processuali con formule che esprimono l'assenza di potere rispetto all'assunzione di una determinata prova, quali: 'non possono essere assunti come testimoni ...'; 'il testimone non può deporre sulle voci correnti nel pubblico'; 'non possono essere utilizzati, neppure con il consenso della persona interessata, metodi o tecniche idonei a influire sulla libertà di autodeterminazione ... In altri casi, il divieto deriva da regole di inclusione dalle quali lo si ricava a contrario; ad esempio, l'art. 266 comma 1 c.p.p. secondo cui 'l'intercettazione di conversazioni o comunicazioni telefoniche e di altre forme di telecomunicazioni è consentita nei procedimenti relativi ai seguenti reati ...' o l'art. 431 c.p.p. relativo agli atti probatori destinati a confluire nel fascicolo del dibattimento, sulla cui base è pronunciata la decisione finale.

Talvolta, il divieto probatorio è contemplato direttamente nella Costituzione, come accade con la regola del contraddittorio nella formazione della prova contenuta nell'art. 111 comma 4 Cost.: da questa regola - enunciabile nella forma 'se $x$ è prova, allora $x$ deve essere formato in contraddittorio' - si ricava deduttivamente per modus tollens, ossia negando il conseguente, che 'se $x$ non è formato in contraddittorio, allora $x$ non è prova'. In tal caso tocca alla legge ordinaria di uniformarsi al divieto probatorio, fissato dalla fonte gerarchicamente superiore, pena l'illegittimità delle disposizioni che lo non rispettino. Altro problema, che si affronterà in seguito, è se il divieto probatorio possa considerarsi implicito nella tutela costituzionale accordata a determinati diritti, al punto da rendere inutilizzabili le prove raccolte in violazione di quei diritti.

\section{Prove illegittimamente acquisite: a) 'Acquisite' come 'Ammesse'}

Ai sensi dell'art. 191 commi 1 e 2 c.p.p., «Le prove acquisite in violazione dei divieti stabili dalla legge non possono essere utilizzate/L'inutilizzabilità è rilevabile d'ufficio in ogni stato e grado del procedimento».

Nel progetto preliminare del codice di procedura penale la disposizione, alludendo a prove 'ammesse' in violazione dei divieti stabiliti dalla legge, risultava chiaramente volta a sanzionare esclusivamente la violazione dei divieti probatori. Nel testo definitivo la parola 'ammesse' è stata sostituita 
con 'acquisite', espressione alquanto ambigua, che ha dato luogo a due distinte interpretazioni; ed è, anzi, verosimile che la sostituzione sia stata scelta a bella posta, allo scopo di lasciare aperta la possibilità di una duplice lettura.

La prima interpretazione, sostenuta principalmente da Franco Cordero $^{27}$, intende 'acquisite' come 'ammesse', con la conseguenza di limitare l'inutilizzabilità alla violazione dei divieti probatori, ossia alle prove inammissibili. Così inteso, l'art. 191 c.p.p. esprime una mera tautologia, assumendo un valore essenzialmente pedagogico ${ }^{28}$ : la prova ammessa contro i divieti stabiliti dalla legge è ... inammissibile, quindi inutilizzabile. Viceversa, la violazione delle regole previste per l'assunzione di una prova in sé ammissibile è causa di invalidità solo in quanto lo preveda espressamente la legge, restando altrimenti fonte di semplice irregolarità.

Chiare le conseguenze in tema di rapporti tra perquisizione e sequestro e di indebita rivelazione del segreto professionale o d'ufficio. Il sequestro di cose pertinenti al reato a seguito di una perquisizione illegittima è valido perché è lo stesso art. 253 c.p.p. a stabilire che «l'autorità giudiziaria dispone il sequestro», id est l'acquisizione al processo, «del corpo del reato e delle cose pertinenti al reato necessarie per l'accertamento dei fatti», senza subordinarlo ad altra condizione.

Inutile invocare l'art. 191 c.p.p., perché in forza dell'art. 253 c.p.p. ogni cosa pertinente al reato è, tramite il sequestro, legittimamente acquisita al processo. L'illegittima acquisizione implica una prova non ammessa dalla legge, quale uno scritto anonimo, una testimonianza

27 Prove illecite nel processo penale [1961], ora in Tre studi sulle prove penali, cit., 177. V. inoltre, dello stesso A., per il codice abrogato, Procedura penale, 9 ed., Giuffrè, Milano, 1987, 479 s., nonché, per quello vigente, Procedura penale, 9 ed., Giuffrè, Milano, 2012, 628. Nello stesso senso v., fra gli altri, P. Ferrua, La prova nel processo penale, I, Struttura e procedimento, II ed., Torino, 2017, 251 s.; B. Lavarini, Segreto d'ufficio e inutilizzabilità della prova, in Dir. pen. proc., 2004, 900 s.; G. Lozzi, Lezioni di procedura penale, 13 ed., Torino, 2018, 192 s.; A. Scella, Prove penali e inutilizzabilità. Uno studio introduttivo, Giappichelli, Torino, 2000. Per un quadro delle diverse opinioni, A. Camon, Le prove, in AA.VV., Fondamenti di procedura penale, Cedam, Milano, 2019, 301 s. Ad evitare equivoci, va ricordato che condizione generale di ammissibilità delle dichiarazioni rese nel processo è la libertà del volere: qualsiasi lesione della libertà di autodeterminazione implica l'inesistenza giuridica della dichiarazione resa.

Cordero, Procedura [2012], cit., 629 s. 
estorta; o, ancora, una dichiarazione raccolta fuori dal contraddittorio e acquisita al dibattimento fuori dai casi in cui ne è ammessa l'utilizzazione. In quest'ultimo caso - che riguarda principalmente le informazioni testimoniali ricevute dagli organi inquirenti - si parla di 'inutilizzabilità fisiologica' perché l'atto è utilizzabile in sede di indagini preliminari ed è conforme alla fattispecie che lo disciplina; ma, rispetto al dibattimento, si è in presenza, come nelle altre ipotesi, di una prova inammissibile.

Analoghe le conclusioni in tema di rivelazione spontanea del segreto professionale o d'ufficio, in rapporto alla quale non si rinviene nel codice di rito alcun divieto probatorio. Per il segreto professionale, l'art. 200 c.p.p. dispone che i titolari «non poss[a]no essere obbligati a deporre» (se lo fossero, naturalmente, la prova sarebbe inammissibile, perché acquisita contro i divieti stabiliti dalla legge); per il segreto d'ufficio, invece, l'art. 201 c.p.p. contempla «l'obbligo di astenersi dal deporre». È probabile che, con la seconda formula, i compilatori del codice intendessero porre un divieto probatorio; ma, come gli apprendisti stregoni, non sono riusciti ad introdurlo. L'essere 'obbligati a non deporre' non equivale a 'non possono deporre'. La prima figura attiene ad un profilo esclusivamente soggettivo; la seconda ad un oggettivo divieto probatorio. 'Obbligati' dalla legge penale a non deporre sono tanto i titolari del segreto professionale quanto quelli del segreto d'ufficio; ma né per gli uni né per gli altri è contemplato un divieto probatorio nel senso proprio della parola, il quale implicherebbe formule - come per l'appunto 'non possono deporre' o ' essere assunti come testimoni' - tali da risolversi, per il giudice, nell'assenza del potere di assumere la deposizione. La spontanea rivelazione di un segreto professionale o d'ufficio costituisce, perciò, una prova validamente assunta.

\section{Segue: в) acquisite come 'ottenute'}

La seconda interpretazione - sostenuta in primis da Massimo Nobili $^{29}$ - intende 'acquisite' come 'ottenute' o 'scoperte' o 'raccolte',

29 M. Nobili, La nuova procedura penale, Clueb, Bologna, 1989, 157 s.; R. Gambini, Perquisizioni, sequestri, esclusione probatoria: interpretazioni attuali e prospettive de jure condendo, in Diritto penale e processo, 2005, 1289 s.; L.P. Comoglio, Perquisizione illegittima ed inutilizzabilità derivata delle prove acquisite con susseguente sequestro, in Cass. pen., 1996, 1547 s. 
con la conseguenza di rendere inutilizzabile ogni prova le cui modalità di ricerca e di assunzione non siano state rigorosamente osservate o che risulti legata da un nesso causale ad una qualsiasi violazione di legge (anche penale sostanziale 0 , persino, civile). In questa prospettiva l'illegittimità della perquisizione invalida il sequestro, determinando l'inutilizzabilità dei reperti: non è dubbio, infatti, che tra l'una e l'altro sussista un nesso di dipendenza causale, dato che il materiale sequestrato è stato 'scoperto' grazie alla perquisizione. Altrettanto vale per la spontanea rivelazione del segreto professionale o d'ufficio: la testimonianza risulta, infatti, 'ottenuta' attraverso la commissione di un reato.

Così inteso, l'art. 191 c.p.p., lungi dal ridursi ad una mera tautologia, assume un rilievo centrale, sviluppando l'effetto dirompente di sanzionare con l'inutilizzabilità del risultato qualsiasi irregolarità realizzata nel procedimento probatorio; ma, in compenso, diventano superflue le specifiche previsioni di invalidità stabilite in materia probatoria da singole disposizioni, in quanto già deducibili dalla norma generale.

Questa prospettiva, sulla scia dell'ordinamento angloamericano, viene spesso designata come teoria dei frutti dell'albero avvelenato. Ma è una metafora ingannevole nella parte in cui lascia intendere che la perquisizione produca le cose sequestrate, come l'albero i suoi frutti. Le cose rinvenute non sono né il frutto né il prodotto della perquisizione illegittima, ma semplicemente il suo esito occasionale; la perquisizione in sé, per illegittima che sia, non altera né contamina ciò che si scopre, la cui esistenza è indipendente dall'attività euristica ${ }^{30}$. Naturalmente è possibile che i reperti siano manipolati da chi effettua la perquisizione o ivi dolosamente collocati, ma abusi ed illegalità del genere possono realizzarsi anche in sede di perquisizioni regolarmente autorizzate.

\section{La PROSPETTIVA COSTITUZIONALE}

Sul piano strettamente codicistico, a favore dell'interpretazione che circoscrive l'ambito sanzionatorio dell'art. 191 c.p.p. ai divieti probatori, ossia alle prove inammissibili, milita un semplice rilievo: in

30 V. Bozio, La prova atipica, cit., 86, nota 121. 
tanto una prova può ritenersi 'illegittimamente acquisita' ai sensi dell'art. 191 c.p.p. in quanto si riscontri un vizio nel mezzo acquisitivo, il quale nei casi ipotizzati è rappresentato dal sequestro del materiale rinvenuto o dalla deposizione spontaneamente resa sul segreto. Ma, poiché il sequestro è subordinato alla sola condizione che la cosa sia pertinente al reato e nessuna disposizione limita il potere di assumere la testimonianza di chi deponga spontaneamente su un segreto, non si vede come si possa concludere per l'illegittima acquisizione della prova ai sensi dell'art. 191 c.p.p.

Più complesso appare il discorso se svolto nella prospettiva dei principi costituzionali, che lasciano aperto un interrogativo, variamente affrontato e risolto in dottrina: può ritenersi o no compatibile con le disposizioni della Costituzione e della Convenzione europea, relative alla tutela della libertà personale e del domicilio, una disciplina che riconosca piena validità al sequestro delle cose rinvenute in una perquisizione illegittima? Ed altrettanto dicasi in rapporto alla deposizione spontaneamente resa in violazione del segreto professionale o d'ufficio.

A differenza di quanto accade per il contraddittorio, dove la regola di esclusione probatoria per le dichiarazioni raccolte nel segreto è deduttivamente ricavabile dal 4 comma dell'art. 111 Cost., né la Costituzione né la Convenzione europea, pur tutelando entrambe il domicilio e la libertà personale - definiti inviolabili dalla Costituzione dettano espressi divieti probatori in questa materia. Questo, peraltro, non esclude che qualcuno possa ritenerli necessari per l'effettività delle garanzie costituzionali o convenzionali, sino a considerarli impliciti in quei testi. Ma, trattandosi pur sempre di divieti non contemplati da una norma processuale della Costituzione o della Convenzione, la via corretta per il giudice, che intenda affermarli, non è di immetterli a viva forza nel tessuto codicistico con acrobatiche interpretazioni creative, ma di sollevare la questione di legittimità costituzionale ${ }^{31}$.

31 Sulla possibilità per il giudice di estromettere direttamente dal processo le c.d. prove incostituzionali, v. A. Camon, Le prove, cit., 303 s. A nostro avviso occorre evitare di confondere, sotto la denominazione di 'prova incostituzionale', due fenomeni ben distinti. Un conto è la prova acquisita in violazione di una regola di esclusione probatoria stabilita da una norma processuale della Costituzione, come, ad esempio, il quarto e il quinto comma dell'art. 111 Cost.; altro è la prova alla cui origine si riscontri la violazione di un qualsivoglia diritto costituzionalmente protetto, come la libertà personale o la tutela 
La Corte costituzionale, nella motivazione di un lontano precedente (sentenza n. 34 del 1973), era parsa in qualche modo favorevole a ritenere sanzionabile in sede processuale la violazione dei precetti della Costituzione, quando aveva avvertito «il dovere di mettere nella dovuta evidenza il principio secondo il quale attività compiute in dispregio dei fondamentali diritti del cittadino non possono essere assunte di per se a giustificazione ed a fondamento di atti processuali a carico di chi quelle attività costituzionalmente illegittime abbia subito»; ma quell'obiter dictum, contenuto in una sentenza di rigetto, non ha avuto seguito ed è rimasto una mera affermazione di principio. Una dichiarazione di illegittimità costituzionale o una riforma legislativa, volte a negare valore alle prove raccolte in violazione dei diritti costituzionali (le cd prove incostituzionali), rappresenterebbero una sorta di via intermedia tra le opposte e più radicali letture dell'art. 191 c.p.p., messe a confronto nel paragrafo precedente.

Dal canto suo, la Corte europea dei diritti dell'uomo ha fornito, in varie occasioni, spunti per un'analoga interpretazione delle disposizioni convenzionali; e, di recente, ha censurato una perquisizione svolta in assenza di un qualsivoglia controllo giurisdizionale, rammentando «di avere ammesso che, in alcune circostanze, il controllo della misura contraria all'articolo 8 effettuato dai giudici penali fornisce una riparazione adeguata per l'interessato, dal momento che il giudice procede a un controllo effettivo della legittimità e della necessità della misura contestata e, se del caso, esclude dal processo penale gli elementi di prova raccolti (Panarisi c. Italia, n. 46794/99, parr. 76 e 77, 10 aprile 2007, Uzun c. Germania, n. 35623/05, parr. 71 e 72, CEDU 2010 (estratti), e Trabajo Rueda c. Spagna, n. 32600/12, par. 37, 30 maggio 2017)» (il corsivo è mio) ${ }^{32}$.

del domicilio. Se nel primo caso si può ipotizzare una diretta applicazione della norma costituzionale, nel secondo, quando l'inutilizzabilità non sia deducibile dal codice di rito, il giudice che intenda affermarla deve sollevare questione di legittimità costituzionale: v. in tal senso F. Caprioli, Colloqui riservati e prova penale, Giappichelli, Torino, 2000, 236 s.; F. Cordero, Procedura [2012], cit., 639; N. Galantini, L'inutilizzabilità della prova nel processo penale, Cedam, Padova, 1992, 204 s. Sulla categoria della 'prova incostituzionale' v. P. Tonini - C. Conti, Il diritto delle prove penali, 2 ed., Giuffrè, Milano, 2014, 104 s.

32 Corte Europea dei diritti dell'uomo 27 settembre 2018, Brazzi c. Italia (Ricorso n. 57278/11), in Questione giustizia (on line), 15 gennaio 2019, con nota di D. Cardamone, La sentenza della Cedu Brazzi c. Italia: sono arbitrarie le perquisizioni disposte dall'Autorità giudiziaria? 


\section{UN'ECCEPIBILE SENTENZA COSTITUZIONALE DI INAMMISSIBILITÀ}

Con ordinanza del 12 dicembre 2017 il Tribunale di Lecce sollevava «la questione di illegittimità costituzionale dell'art. 191 c.p.p., per contrasto con gli articoli 2, 3, 13, 14, 24, 97 comma 3 e 117 Cost. (quanto a quest'ultima norma, con riferimento ai principi di cui all'art. 8 della Convenzione europea dei diritti dell'uomo), nella parte in cui non prevede che la sanzione dell'inutilizzabilità ai fini della prova riguardi anche gli esiti probatori, ivi compreso il sequestro del corpo del reato o delle cose pertinenti al reato, degli atti di perquisizione ed ispezione compiuti dalla p.g. fuori dei casi tassativamente previsti dalla legge o comunque non convalidati dall'A.G. con provvedimento motivato, nonché la deposizione testimoniale in ordine a tali attività».

Si apriva, così, un'ottima occasione per sciogliere un ricorrente interrogativo: la Corte avrebbe finalmente detto con chiarezza se le garanzie sulla inviolabilità della libertà personale e del domicilio richiedessero o no per la loro effettività, accanto alle sanzioni penali e disciplinari, la messa al bando delle prove raccolte in loro spregio.

Attesa delusa perché la Corte costituzionale con sentenza n. 219 del 2019 ha dichiarato inammissibile la questione: il giudice a quo avrebbe avanzato «una richiesta fortemente 'manipolativa', pretendendo di desumere l'automatica inutilizzabilità degli atti di sequestro, attraverso il ‘trasferimento' su di essi dei vizi che affliggerebbero gli atti di perquisizione personale e domiciliare dai quali i sequestri sono scaturiti».

Detto con il massimo rispetto, dissentiamo fermamente dalla dichiarazione di inammissibilità.

La Corte concorda sulla circostanza che l'interpretazione restrittiva dell'art. 191 c.p.p. assunta dal giudice di merito a base del quesito di legittimità costituisca diritto vivente; e, anzi, ritiene che sia l'unica ragionevolmente sostenibile a fronte del dato testuale. Proprio per questo vi sarebbero stati, a nostro avviso, tutti i presupposti per addivenire ad una

Sul regime delle prove assunte in violazione delle norme convenzionali v. A. Cabiale, I limiti alla prova nella procedura penale europea, Cedam, Milano-Padova, 2019, $133 \mathrm{~s}$. Sulla difficoltà di desumere regole di esclusione probatoria dall'art. 8 CEDU, v. M. Daniele, Indagini informatiche lesive della riservatezza. Verso un'inutilizzabilità convenzionale?, in Cass pen., 2013, 367 s. 
decisione sul merito, di accoglimento o di rigetto a seconda dell'indirizzo seguito. Il rigetto della questione come infondata o manifestamente infondata - che, come vedremo, viene in qualche modo avvallato da un obiter dictum contenuto nella motivazione dell'inammissibilità - avrebbe semplicemente accertato la compatibilità della disciplina vigente con la Costituzione, senza minimamente imporla come una scelta necessaria; quindi, senza pregiudicare la libertà del legislatore di intervenire in futuro, estendendo la inutilizzabilità anche alle cose illecitamente rivenute ${ }^{33}$.

Quanto all'eventuale decisione di accoglimento, non si vede cosa vi sarebbe stato di 'manipolativo' o comunque di estraneo ai poteri della Corte nel dichiarare illegittimo l'art. 191 c.p.p. nella parte in cui non vieta di utilizzare i reperti rinvenuti a seguito di una perquisizione illegittima; o nel dichiarare illegittimo, in via principale o derivata, l'art. 253 c.p.p. nella parte in cui consente il sequestro delle cose pertinenti al reato anche in caso di perquisizioni illegittime.

La dichiarazione di illegittimità sarebbe discesa 'a rime obbligate' dalla ritenuta insufficienza delle sanzioni disciplinari e penali a garantire la tutela del domicilio e della libertà personale in sede processuale. A ritenere manipolativa una sentenza del genere, lo sarebbero in buona parte le sentenze di accoglimento; e, in ogni caso, lo sarebbe meno delle numerose sentenze additive emanate nel passato (si pensi, ad esempio, a Corte cost. n. 361 del 1998 relativa all'art. 513 c.p.p. o, per citare una decisione successiva a quella in esame, a Corte cost. n. 242 del 2019 relativa al c.d. suicidio assistito, che, per quanto lodevole, ben difficilmente può dirsi imposta 'a rime obbligate' dalle disposizioni costituzionali).

\section{Gli obiter dicta della Corte costituzionale}

Pur dichiarando inammissibile la questione, la Corte costituzionale non rifugge da una serie di obiter dicta ${ }^{34}$, uno dei quali lascia intendere,

${ }_{33}$ Per un auspicio in questo senso, v. G. Spangher, "E pur si muove": dal male captum bene retentum alla exclusionary rules, in Giur. cost., 2001, 2829.

34 Nelle sentenze di inammissibilità gli obiter dicta attinenti al merito si sono resi sempre più frequenti negli ultimi tempi. Un significativo esempio è costituito dalla sentenza Corte cost. n. 132 del 2019 che, dopo avere dichiarato 
in forma abbastanza trasparente, l'infondatezza della questione sollevata. Alludiamo, in particolare, al passo in cui la Corte sostiene «che se è vero quanto afferma il giudice a quo a proposito del fatto che le regole che stabiliscono divieti probatori riposano essenzialmente sulla esigenza di introdurre misure volte anche a disincentivare possibili 'abusi' [...] è altrettanto vero che un simile obiettivo viene in ogni modo perseguito dall'ordinamento attraverso la persecuzione diretta, in sede disciplinare o, se del caso, anche penale, della condotta 'abusiva' che possa essere stata posta in essere dalla polizia giudiziaria».

Perché la Corte, sviluppando questa prospettiva, non ha dichiarato infondata la questione? Perché si è preferito scrivere nella motivazione di una sentenza di inammissibilità ciò che meglio si sarebbe adattato ad una dichiarazione di infondatezza? Non è difficile immaginare i motivi.

Anzitutto, il timore di contraddire quel lontano precedente già menzionato sull'impossibilità di porre a fondamento degli atti processuali «attività compiute in dispregio dei fondamentali diritti del cittadino». Sicuramente ha avuto anche il suo peso la recente sentenza della Corte EDU che ha ritenuto insufficienti le garanzie del nostro ordinamento contro il rischio di perquisizioni arbitrarie, data l'assenza di adeguati controlli sia ex ante sia ex post ${ }^{35}$, accennando alla possibilità che il giudice escluda dal processo le prove raccolte; non si dimentichi che, secondo un assunto della Corte costituzionale, poi parzialmente ritrattato, le garanzie della Convenzione europea dovrebbero essere applicate da tutti i giudici nazionali come interpretate dalla Corte di Strasburgo ${ }^{36}$. Infine, una certa

inammissibile una questione di legittimità costituzionale relativa all'art. 525 c.p.p., si è prodigata in una serie di suggerimenti al legislatore sull'opportunità di introdurre deroghe alla rinnovazione delle prove in caso di rinvio del dibattimento davanti ad un diverso collegio giudicante; il tutto, senza adombrare alcun profilo di illegittimità della disciplina vigente: al riguardo v. i contributi di P. Ferrua - O. Mazza - D. Negri - L. Zilletti, nel Confronto di idee su: La post immediatezza nella nuova giurisprudenza costituzionale (a margine della sentenza n. 132 del 2019), in Arch. pen. (on line), 2019, 2. In una corretta ripartizione dei poteri i suggerimenti della Corte costituzionale al legislatore si giustificano nella stretta misura in cui siano diretti a prevenire o ad eliminare conflitti con i precetti della Costituzione. Corte EDU 27 settembre 2018, Brazzi c. Italia, cit.

36 Così le sentenze 'gemelle' (Corte cost. n. 348 e n. 349 del 2007); l'assunto sul carattere vincolante delle interpretazioni della Corte europea è, a nostro 
influenza va ascritta anche al timore che un'esplicita dichiarazione di infondatezza potesse incoraggiare abusi da parte della polizia giudiziaria nello svolgimento di ispezioni o perquisizioni.

Comprendiamo l'imbarazzo della Corte, stretta tra quei precedenti sull'esigenza di un pieno rispetto nel processo dei diritti fondamentali e una dichiarazione di illegittimità, capace di mandare in fumo prove di essenziale rilevanza per l'accertamento di gravi reati; al punto che la dichiarazione di inammissibilità le è forse parsa la soluzione ideale per un commodus discessus.

Ma a chi, se non alla Corte costituzionale, compete il potere di dire con chiarezza se le garanzie della libertà personale e del domicilio implichino o no, in caso di violazione, l'irrilevanza delle prove così ottenute? Non è una risposta semplice, perché vi sono ragioni dall'una e dall'altra parte, ma è precisamente la risposta che ci si attende dal giudice delle leggi, custode della Costituzione.

Con la dichiarazione di inammissibilità la Corte raggiunge, a modo suo, un doppio risultato: si sottrae alle pesanti critiche di scarsa sensibilità per la lesione dei diritti costituzionali, che sicuramente si sarebbero indirizzate ad una eventuale sentenza di rigetto nel merito; ma, al tempo stesso - anche in forza di quell'obiter dictum sulla sufficienza delle sanzioni penali e disciplinari - paralizza, con un energico effetto dissuasivo, ulteriori eccezioni di legittimità sul medesimo tema.

avviso, improvvido per due motivi. Anzitutto perché lesivo del principio di soggezione del giudice alla sola legge. Poi perché trascura la fondamentale distinzione tra motivazione e dispositivo: il dispositivo, in quanto comando, è senz'altro vincolante, ma la motivazione - dove è contenuta l'interpretazione della legge - in quanto esercizio di ragione e non comando, non esercita alcuna efficacia vincolante, risultando semplicemente più o meno persuasiva. Sul tema v., da ultimo, P. Ferrua, Il giusto processo tra governo della legge ed egemonia del potere giudiziario, in Dir.pen.proc., 2020, 5 s., e, in precedenza, ID. , Il contraddittorio nella formazione della prova a dieci anni dalla sua costituzionalizzazione: il progressivo assestamento della regola e le insidie della giurisprudenza della Corte europea, in Arch. pen., 2008, n. 3, 29. In senso adesivo verso le sentenze 'gemelle' v., invece, G. Ubertis (La "rivoluzione d'ottobre" della Corte costituzionale e alcune discutibili reazioni, in Cass. pen., 2012, 20 s.; Ancora sull'efficacia della giurisprudenza di Strasburgo, in Dir.pen. proc., 2013, 863 s.; La Corte di Strasburgo quale garante del giusto processo, ivi, 2010, $372 \mathrm{~s}$.), il quale $\mathrm{mi}$ ha ripetutamente criticato con una passione davvero straordinaria. 
Se la richiesta del giudice a quo era, come afferma la Corte, fortemente manipolativa, la possibilità di eccepire l'illegittimità dell'art. 191 c.p.p. quanto ai rapporti tra perquisizione e sequestro - ma per analoghe ragioni anche in ordine alla violazione del segreto professionale o di ufficio - di fatto è persa per sempre: non perché si sia accertata la legittimità della disciplina vigente, ma perché la stessa fin de non recevoir verrebbe opposta a nuove richieste, inevitabilmente destinate ad essere ritenute 'manipolative'.

La discussione proseguirà in dottrina; e, tutto lo lascia pensare, anche nella stessa giurisprudenza dove, come già accaduto, qualche giudice, in dissenso dall'indirizzo dominante e sotto l'alibi della 'prova incostituzionale', troverà modo di 'manipolare' - stavolta il termine è appropriato - il testo dell'art. 191 c.p.p., dichiarando inutilizzabili le cose rinvenute in violazione delle regole sulle perquisizioni o le testimonianze spontaneamente rese sul segreto professionale o d'ufficio. Resta il rammarico che sia sfumata una preziosa occasione per dire parole di chiarezza su una questione di grande rilevanza e, da tempo, al centro di accanite discussioni.

\section{Bibliografia essenziale}

Bozio V., La prova atipica, in P. Ferrua-E. Marzaduri-G. Spangher (a cura di), La prova penale, Giappichelli, Torino, 2013, $57 \mathrm{~s}$.

Busetto M.L., Il contraddittorio inquinato, Cedam, Padova, 2009.

Camon A., Le prove, in AA.VV., Fondamenti di procedura penale, Cedam, Milano, $2019,301 \mathrm{~s}$.

Caprioli F., Colloqui riservati e prova penale, Giappichelli, Torino, 2000.

Caprioli F., voce Condanna (dir. proc. pen.), in Enc. dir., Annali, vol. II, t. 1, Giuffrè, Milano, 2008, p. 111 s.

Carnelutti F., La prova civile [1015], ristampa, Giuffrè, Milano.

Comoglio L.P., Perquisizione illegittima ed inutilizzabilità derivata delle prove acquisite con susseguente sequestro, in Cass. pen., 1996, 1547 s.

Cordero F., Procedura penale, IX ed., Giuffrè, Milano, 2012. 
Cordero F., Tre studi sulle prove penali, Giuffrè, Milano 1963.

Daniele M., Regole di esclusione e regole di valutazione della prova, Giappichelli, Torino, 2009.

Di Chiara G., Il canto delle sirene: Processo penale e modernità scientifico-tecnologica: prova dichiarativa e diagnostica della verità, in Criminalia, 2007, 19 s.

Ferrua P., La prova nel processo penale, vol. I, Struttura e procedimento, Giappichelli, Torino, 2015.

Ferrua P., Onere della prova, regola di giudizio e standard probatorio: alla ricerca della perduta proposizione da provare, in Cass. pen., 2020, 2639 s.

Galantini N., L'inutilizzabilità della prova nel processo penale, Cedam, Padova, 1992.

Gambini R., Perquisizioni, sequestri, esclusione probatoria: interpretazioni attuali e prospettive de jure condendo, in Diritto penale e processo, 2005, 1289 s.

Grevi V., Prove, in G. Conso-V. Grevi-M. Bargis, Compendio di procedura penale, 6 ed., Cedam, Padova, 313 s.

Grice P., Logica e conversazione (1989), trad. it., Il Mulino, Bologna, 1993.

Grifantini F., sub art. 188, in Commentario breve al codice di procedura penale, a cura di G. Conso-V. Grevi, Cedam, Padova, 2005, 530.

Iacoviello F. M., La Cassazione penale. Fatto, diritto e motivazione, Giuffrè, Milano, 2013, 432, 632 s.

Lavarini B., Segreto d'ufficio e inutilizzabilità della prova, in Dir. pen. proc., 2004, 900 s.

Nobili M., La nuova procedura penale, Clueb, Bologna, 1989.

Paulesu P., La presunzione di non colpevolezza dell'imputato, 2 ed., Giappichelli, Torino, 2009.

Ricci G.F., Le prove atipiche, Giuffrè, Milano, 1999.

Santoriello C., Il vizio di motivazione tra esame di legittimità e giudizio di fatto, Utet, Torino, 2008.

Scella A., Prove penali e inutilizzabilità. Uno studio introduttivo, Giappichelli, Torino, 2000.

Scomparin L., La tutela del testimone nel processo penale, Cedam, Padova, 2001. 
Spangher G., "E pur si muove”: dal male captum bene retentum alla exclusionary rules, in Giur. cost., 2001, 2829.

Tonini P. - Conti C., Il diritto delle prove penali, 2 ed., Giuffrè, Milano, 2014.

Ubertis G., Processo indiziario e valutazione probatoria, in Diritto\&Questioni pubbliche, 2020/1, giugno, 317 s.

\section{Additional information and author's declarations (scientific integrity)}

Conflict of interest declaration: the author confirms that there are no conflicts of interest in conducting this research and writing this article.

Declaration of authorship: all and only researchers who comply the authorship requirements of this article are listed as authors; all coauthors are fully responsible for this work in its entirety.

Declaration of originality: the author assures that the text here published has not been previously published in any other resource and that future republication will only take place with the express indication of the reference of this original publication; he also attests that there is no third party plagiarism or self-plagiarism.

Dados do processo editorial (http://www.ibraspp.com.br/revista/index.php/RBDPP/about/editorialPolicies)

- Recebido em: 28/02/2021

- Rodada de correções: 04/03/2021

Autor convidado - invited author
Equipe editorial envolvida

- Editor-chefe: 1 (VGV)

- Editoras-associadas: 2 (KK e BC) 


\section{HOW TO CITE THIS ARTICLES (ABNT BRAZIL):}

FERRUA, Paolo. Ammissibilità della prova e divieti probatori. Revista Brasileira de Direito Processual Penal, Porto Alegre, vol. 7, n. 1, p. 215-246, jan./abr. 2021. https://doi.org/10.22197/rbdpp.v7i1.533

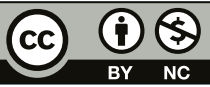

Esta obra está licenciada com uma Licença Creative Commons Atribuição-NãoComercial 4.0 Internacional. 